\title{
Construction and Application of Clothing Pattern Design Model Based on Directed Graph Method
}

\author{
Chao-Jun He ${ }^{\mathrm{a}, \mathrm{b}}$, Bo-An Ying ${ }^{\mathrm{a}, \mathrm{b}, *}, \quad$ Xiao-Feng Wang ${ }^{\mathrm{a}}$, Jing Qi ${ }^{\mathrm{a}}$ \\ a Apparel 83 Art Design College, Xi'an Polytechnic University, Xi'an, Shaanxi 710048, China \\ ${ }^{\mathrm{b}}$ Cooperative Innovational Center for Technical Textiles, Xi'an Polytechnic University, \\ Xi'an, Shaanxi 710048, China
}

\begin{abstract}
At present, the intelligent design of clothing patterns still needs to rely on the experience of the pattern maker, and it cannot liberate the pattern maker from the complicated design work. The efficiency and flexibility of the design of clothing patterns are not high. In order to realize the intelligent pattern design of design knowledge automation, a directed graph method is proposed to construct a clothing pattern design model, and the model is used to analyze the clothing pattern. In view of the characteristics of clothing pattern design, the directed graph method is used to record the pattern design process, and the model of clothing pattern design is constructed. The model is accurately analyzed by decomposing the original model of clothing pattern design into sub-models of each structural line. Application examples verify the feasibility of constructing a pattern design model and application by the directed graph method. The research results show that the pattern design model can clearly express the pattern design process and the valuable experience involved, and can accurately and quickly analyze the pattern. This research can provide a way to extract, collect, process, share and reuse pattern design knowledge for intelligent clothing design, and improve the efficiency of clothing pattern design.
\end{abstract}

Keywords: Directed Graph; Knowledge Automation; Clothing Pattern Design Model

\section{Introduction}

"Made in China 2025" pointed out that intelligent manufacturing is the main direction of China's manufacturing 2025 [1], and knowledge automation is the foundation and the key to the intelligent industry [2]. Knowledge automation mainly refers to the automation of knowledge-based work. Knowledge-based work is the use and creation of knowledge. It is the work that can be completed only by a knowledgeable person or system. It is a creative mental work that produces useful information and knowledge [3].

\footnotetext{
${ }^{*}$ Corresponding author.

Email address: yingba2006@163.com (Bo-An Ying).
} 
At present, there are three main ways to realize the intelligent design of clothing patterns: 1) the use of artificial intelligence technologies; 2) unfolding 3D clothing to form a 2D pattern; 3) basing on the parametric design ideas [4]. In terms of artificial intelligence: Liu Weimin et al. [5] used the BP neural network algorithm to establish the changes on waist and hip size circumference. Through directly using the matching of data, the parametric design of men's waistband, abdomen, and hip modification rules was realized. Preliminarily, it also realized the intelligent pattern design of male tailored trousers. Liu Fang et al. [6] used human dimension data as the input vector and the sample size of the fitted female suits as the output vector. By establishing a BP neural network model, the nonlinear mapping relation between the body size data and the sample size data of the suit was studied to realize the automatic generation from the body size to the pattern size of the tailored suit.

In terms of unfolding 3D clothing to form a 2D pattern, Zhou Hengji [7] constructed a personalized 3D digital human mannequin for legs accords, with the characteristics of the human body, and flatten it to get a 2D vector. The research has been conducted on the generation of rules from the 2D vector to the female trousers prototype. Yuko Mesuda et al. [8] proposed a method of virtual draping by representing the actual draping process, i.e., a pattern is made from cloth model through mapping.

In terms of parametric design, in Ye Qinwen's research [9], the double arc was used to fit the garment pattern contour curve and in combination with the parameterization function of Auto CAD, the parameterized curve constraint model was constructed. Based on this, a parameterized garment pattern was established. In addition, the paper pattern generation efficiency of personalized clothing was improved using this method. Zhang Linli et al. [10] developed parametric patternmaking software for the back body of a shirt based on the Mat Lab platform of software development. The software promoted the response capability of apparel customization.

In other aspects, Hyun-Sook Han et al. [11] developed an automated custom-pattern-making system using the width-height independent grading method for semi-customized clothing. Once the basic-size pattern and grading value are prepared, the width-height independent grading system can quickly generate numerous patterns of different proportions by combining waist girth and height measurements. Adrian R.G. Harwood et al. [12] introduced JBlockCreator, an application and extensible API for the automatic drafting of custom pattern blocks from body measurement data. JBlockCreator provides plotting tools for displaying complex measurement data in a manner which facilitates the study of pattern drafting theory and helps towards the development of new relationships between measurements, consequently improving the situation in practice.

The current artificial intelligence and parametric design methods are well suitable for the pattern intelligent design of fixed clothing styles, such as men's shirts. For the changeable clothing style design, the pattern still needs to be adjusted by pattern designers. The method of unfolding $3 \mathrm{D}$ clothing to form a 2D pattern has high requirements on the operator's technical and artistic accomplishments, so only by relying on the experience of the designer can we design a good clothing pattern. Therefore, although the existing methods have realized the intelligent design of the clothing pattern to a certain extent, it cannot liberate the pattern designer from the complicated design work and in addition, still relies highly on the experience of the patternmaker. To realize the intellectualization of pattern design knowledge, a directed graph method is used to record the pattern design process and analyze the pattern, hoping to reveal the design experience of the designers and provide a way for the automation of pattern design knowledge. 


\section{Principles of the Directed Graph Method to Construct Clothing Pattern Design Model}

\subsection{Design Features of Clothing Pattern}

The clothing pattern design is to first determine one point, then determine another point based on clothing knowledge (pattern-making formula, size formula, empirical data, etc.), and then determine more points. Two points are connected to form a line or multiple points are connected to form a curve or surface. Repeat this process continuously and finally form a clothing pattern [13].

\subsection{Characteristics of Directed Graph Method}

The graph is a graphic. The graphic uses points to represent things, and lines to describe relationships between things abstractly, regardless of the location of points or the length of lines [14]. If each line of the graphic is directed, it is called a directed graph [14]. The directed graph method uses directed graphs to analyze and solve problems. The directed graph model uses the concept of graph theory to model the system. The nodes in the model represent the constituent units or variables of the system, and the directed edges between the nodes reflect some kind of interactive relationship of the system [15].

\subsection{Principles of Constructing Clothing Model Design Model}

From the perspective of artificial intelligence, the problem of pattern design is the problem of constraint satisfaction, and the details of the design object can be acquired by providing the description of constraints in many aspects such as function, structure, and process [16]. Thus, the directed graph model can be used to analyze the knowledge of the pattern design. Furthermore, the constraint relationship between the point and line elements that constitute the clothing pattern can be recorded. It can intuitively and clearly express the process of pattern design and the valuable experience of pattern designers involved. Most of these experiences are personal knowledge of the designers and are difficult to express or share.

\section{Model Construction Rules for Clothing Pattern Design}

\subsection{Pattern Design Process Pre-processing}

Due to the differences in the pattern-making methods and language expressions adopted by different designers, before the construction of the model of the clothing pattern design process, the following aspects of the pattern design process need to be pre-processed:

1) All points and lines appearing in the pattern design process must be named. Structured lines that constitute the pattern must be named one by one according to the process description.

2) If there are constraints (values, formulas, geometry relationships, etc.) in the process flow of generating points and lines, all must be specified. 


\subsection{Pattern Design Model Construction Rules}

The clothing pattern is composed of graphic elements such as points, lines, and surfaces. The design process of clothing pattern involves connecting points into lines, the lines into surfaces. Eventually, they form a garment pattern, and the formation of the points and lines are constrained by clothing knowledge (such as pattern-making formulas, human body size, empirical data, geometric relationships). In the research, the directed graph model was used to record all points and line elements and their interrelationships in the pattern making process.

According to the definition and theoretical method of a directed graph, the constituent elements in the directed graph model of clothing model design are defined [17]: The definition of a directed graph is that all edges have directions, and all edges of the graph are composed of directed edges. This kind of graph is called a directed graph. In the process of pattern making, the point and line of the pattern corresponding to the vertex of the directed graph, and the parameter relationship between the pattern making elements corresponds to the edge of the directed graph. According to the pattern-making elements and the parameter relationship between the elements, the generation sequence of points and lines is connected to the direct relationship of directed edges. Then the directed graph model of the clothing pattern design is obtained. In the model, circular, rectangular and diamond nodes, and directed edges are used to represent the element information of "points", "auxiliary lines", "structural lines", and the directional relationship of "the process flow of pattern making" information. Moreover, the nodes and directed edges are the constituent elements of the directed graph model of the garment pattern design.

1) Rules for the generation of circular nodes

- It can be generated by a circular node moving by a numerical value or formula;

- It can be generated by a circular node and a rectangular node (that is, the point moves along the line to generate a new point);

- It can be generated by two rectangular nodes (that is, the intersection of auxiliary lines);

- It can be generated by a rectangular node (that is, the equal-dividing point of the auxiliary line).

2) Rules for the generation of rectangular nodes

- It can be generated by a circular node (that is, a horizontal or vertical line made through the point, or a circle made with the point as its center and a given radius);

- It can be generated by a rectangular node (that is, a parallel line of the auxiliary line);

- It can be generated by a circular node and a rectangular node (that is, through the point to make an angle, level or vertical line of the auxiliary line);

- It can be generated by two or more circular nodes (that is, straight lines or curves passing through these points).

3) Rules for the generation of diamond nodes

- It can be generated by two or more circular nodes;

- It can be generated by a circular node and a rectangular node (that is, the generated structural line is a parallel or vertical line of the auxiliary line, and the generated line passing through the point, or it has a certain distance from the point). 


\section{Application Example of Clothing Pattern Design Mod- els}

\subsection{Construction of Donghua Menswear Prototype Design Model}

\subsubsection{Pretreatment of Clothing Pattern Design Process}

China Donghua menswear Prototype design process was exemplified by this article. The size data used in the pattern making process is shown in Table 1 below, and the human body data is the data of the male model named Martin in CLO 3D software.

Table 1: Explanation of data in Donghua menswear prototype design process

\begin{tabular}{llll}
\hline No. & Data name & Data variable name & Data \\
\hline 1 & Height & $\mathrm{H}$ & $188 \mathrm{~cm}$ \\
2 & Bust girth & $\mathrm{B}$ & $96.5 \mathrm{~cm}$ \\
3 & Back width & $\mathrm{B} 1(0.15 \mathrm{~B}+5.6)$ & $20.075 \mathrm{~cm}$ \\
4 & Bust width & $\mathrm{B} 2(0.15 \mathrm{~B}+4.5)$ & $18.975 \mathrm{~cm}$ \\
5 & Back neck point to waist & $\mathrm{BAL}$ & $48.58 \mathrm{~cm}$ \\
6 & Breast height & $\mathrm{H} 10.1 \mathrm{H}+9$ & $27.8 \mathrm{~cm}$ \\
7 & Back shoulder angle & $\mathrm{A} 1$ & $22^{\circ}$ \\
8 & Front shoulder angle & $\mathrm{A} 2$ & $18^{\circ}$ \\
9 & Back Neck Width & $\mathrm{C} 1(0.07 \mathrm{~B}+2)$ & $8.755 \mathrm{~cm}$ \\
10 & Back Neck Vertical Height & $\mathrm{C} 2(\mathrm{C} 1 / 3)$ & $2.92 \mathrm{~cm}$ \\
11 & Front Neck Width & $\mathrm{C} 3 \mathrm{C} 1-0.3)$ & $8.455 \mathrm{~cm}$ \\
12 & Front Neck Vertical Height & $\mathrm{C} 4(\mathrm{C} 1+0.5)$ & $9.255 \mathrm{~cm}$ \\
13 & Back Float remainder & $\mathrm{C} 5(\mathrm{~B} / 40-0.4)$ & $2.013 \mathrm{~cm}$ \\
14 & Front Float remainder & $\mathrm{C} 6 \mathrm{~B} / 40)$ & $2.413 \mathrm{~cm}$ \\
\hline
\end{tabular}

The pre-processing should be prepared for the design process of the prototype. The drawing of the back-center line is illustrated by taking point BW as the starting point and vertically up BAL to point BNP. After connecting point BNP and point BW, the back center line L_BCL was completed. In the process, all points and lines were named (point BNP, point BW, line L_BCL), and the constraint conditions between two points were indicated (value BL).

The following eight methods of determining points and lines are involved in the design process of the prototype:

a. A new point was achieved by a point moved a certain distance;

b. A new point was generated by a point moved a certain distance along a line;

c. A new point was achieved by taking the equal points of a line segment;

d. A point can be determined by the intersection of two lines;

e. A straight line, line segment or curve can be completed by connecting 2 or more points;

f. A new line can be drawn by making a parallel or vertical line of a line through a point; 
g. A new line can be completed by making an angled line of a line through a point on the line.

The design process of some prototype structural lines in Table 2 was used to explain the above 8 methods.

Table 2: Part of the prototype structural lines design process

\begin{tabular}{|c|c|c|}
\hline No. & Structural line & The process of the prototype structural line design (Unit: centimeter) \\
\hline 1 & Back center line & $\begin{array}{l}\text { It takes point } \mathrm{BW} \text { as the starting point and vertically up BAL to point BNP. } \\
\text { After connecting the point BNP and point BW, the back center line L_BCL is } \\
\text { completed. }\end{array}$ \\
\hline 2 & Side seam line & 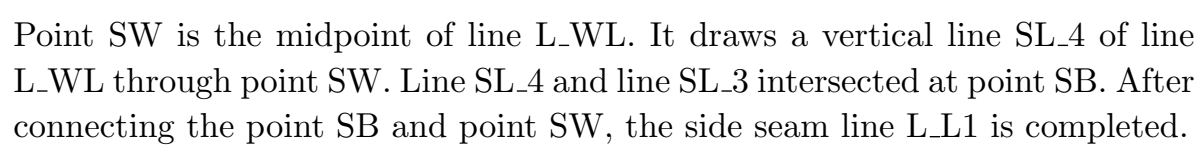 \\
\hline 3 & Front shoulder line & $\begin{array}{l}\text { Straight-line } \mathrm{SL} \_11 \text { is drawn by passing point } \mathrm{P} 13 \text { and with an angle of } \mathrm{A} 1(22 \mathrm{~b}) \\
\text { to line } \mathrm{SL} \_2 . \mathrm{L}_{P 2-P 6} \text { is the measured length of the back shoulder line. Point } \\
\mathrm{P} 16 \text { is drawn by moving point } \mathrm{P} 13 \text { to the right by } \mathrm{L}_{P 2-P 6} \text { along line SL_11. } \\
\text { The front shoulder line } \mathrm{L} \_\mathrm{L} 5 \text { was completed by connecting point } \mathrm{P} 13 \text { and point } \\
\text { P16. }\end{array}$ \\
\hline
\end{tabular}

The completed structure diagram of the prototype is shown in Fig. 1.

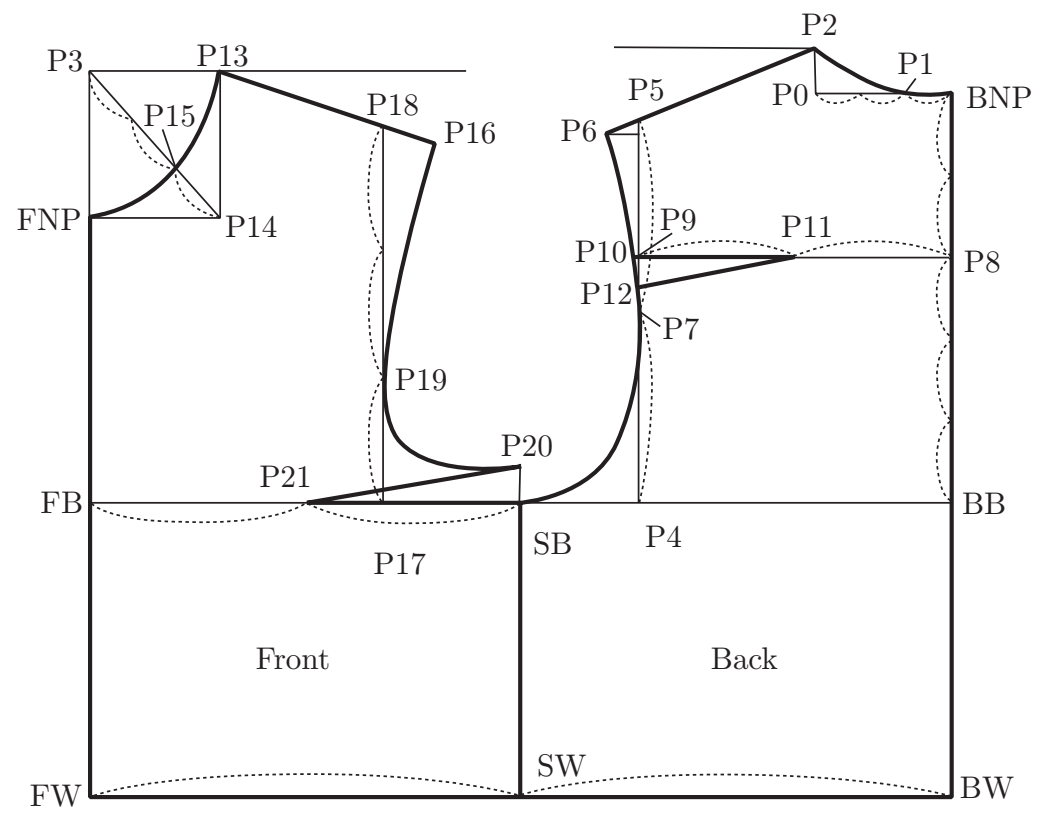

Fig. 1: Structure diagram of Donghua menswear prototype

\subsubsection{Donghua Menswear Prototype Design Model Construction}

According to the processed drawing steps and the construction rules of the nodes of a directed graph, by first determining a circular node, and then determining the position of the second node according to the specific constraints between the second node and the first node, connect the two nodes with directed edges and mark the constraints on the directed edges. After, determine the 
position of the third node according to the constraints of the third node and the second node. Through gradually adding constraints in this way, all the nodes that constitute a pattern are drawn, thus forming the directed graph model of a pattern.

For example, point P1 horizontally moved "a" to the left to point P2, line L1 was completed by connecting point $\mathrm{P} 1$ and point $\mathrm{P} 2$, and drew a vertical line L2 of line L1 through point P2, and point P3 was drawn by moving point P2 to the right by "b" along line L2. The position of circular node P1 is determined by the specific constraints between P1 and P2 nodes. First, connect the two nodes with directed edges, and mark the constraint on the directed edge. Then rectangular node L1 is determined by node P1 and node P2. Gradually adding constraints in this way, we can determine all the nodes: P1, P2, L1, L2, P3. The completed directed graph model is shown in Fig. 2. In the figure below the characters on the directed edges represent constraints between both nodes ends of the directed edge. We use specific characters to express specific meanings and to make the model more concise: " $\mathrm{x}$ " indicates horizontal movement, " $\mathrm{x}$ " indicates movement along a line, "y" indicates vertical movement, "-" indicates leftward or upward movement, "PL" indicates parallel, and "VL" indicates vertical. And the fractions indicate the division point of the line segment. The denominator indicates how many equal divisions the line segment is, and the numerator indicates the number of division points from top to bottom or left to right.

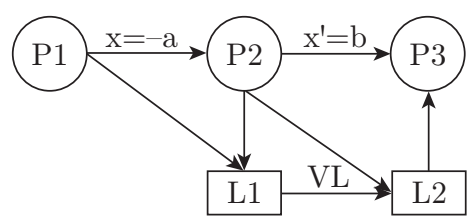

Fig. 2: Example of a directed graph model drawing

Instead of dividing it into two pieces, this article built the directed graph model of the design process of the prototype as a whole, because the front and back pattern of the prototype are closely related and the structure is not very complex. The pattern design model of the prototype was drawn according to the above-mentioned method, as shown in Fig. 3 below. In Fig. 3 below, the part above the red line is the design process of the back pattern of the prototype, and the part below the red dotted line is the design process of the front pattern.

\subsection{Analysis of the Fit of Donghua Menswear Prototype}

Donghua menswear prototype was drawn based on the measured human body data and was imported into the CLO 3D software for virtual sewing. Then the prototype was worn on the virtual model for virtual fitting, and the pattern was set to be transparent to observe the quantity of space distribution between the prototype and the human body. The try-on effect was shown in Fig. 4.

It can be seen from Fig. 4 that the prototype collar line has a large deviation from the model neck baseline, the prototype shoulder exceeds the position of the model's shoulder point, and the bust line of the prototype slightly deviated from the model's bust line. The part of the prototype above the bust line fits Good, the gaps are evenly distributed. And the waist of the prototype is level, basically flush with the model's waistline.

The complete design process of a clothing pattern is complicated. For this reason, the original problem of pattern design can be broken up into subproblems generated by each structural line 


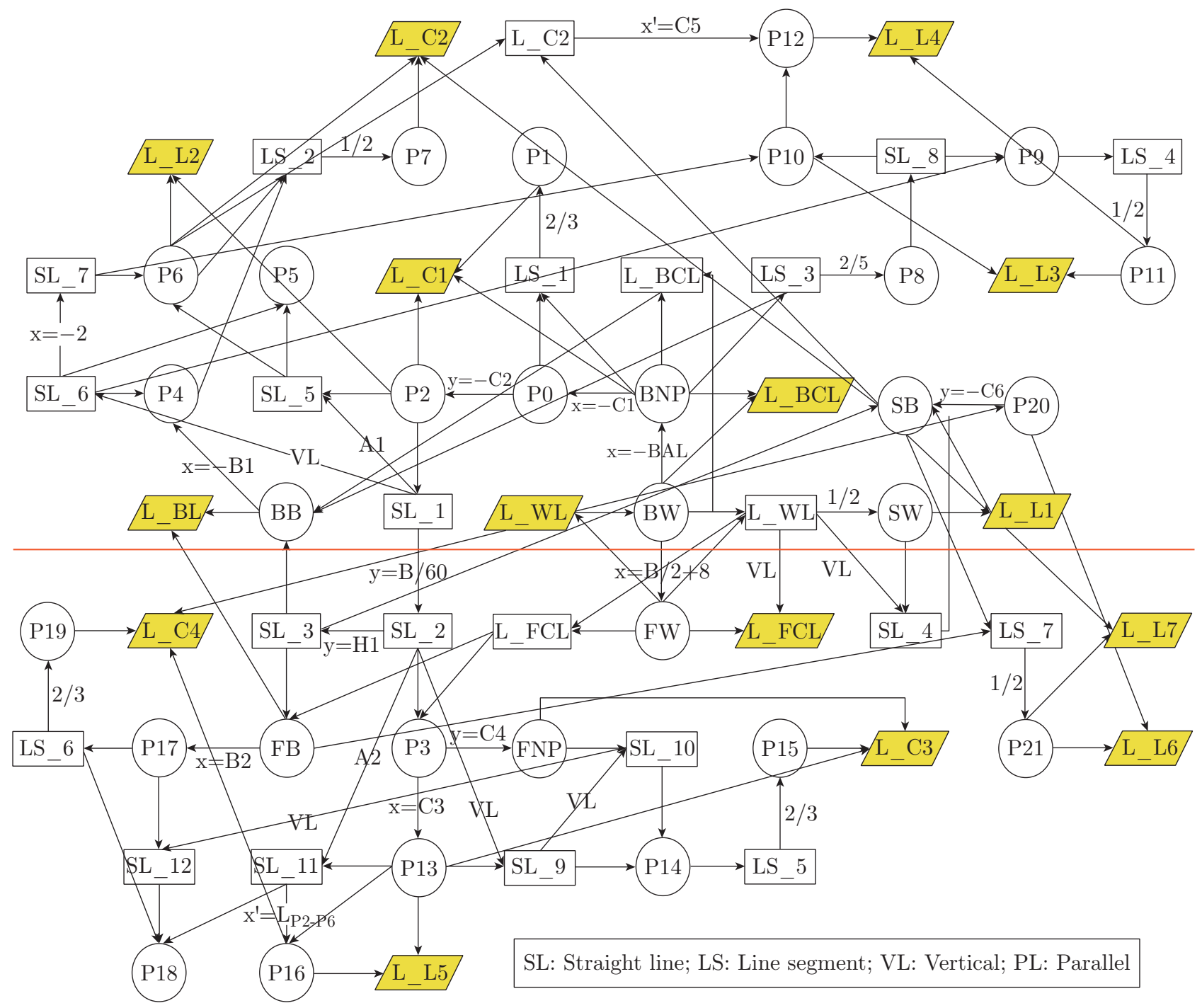

Fig. 3: Donghua menswear prototype design model

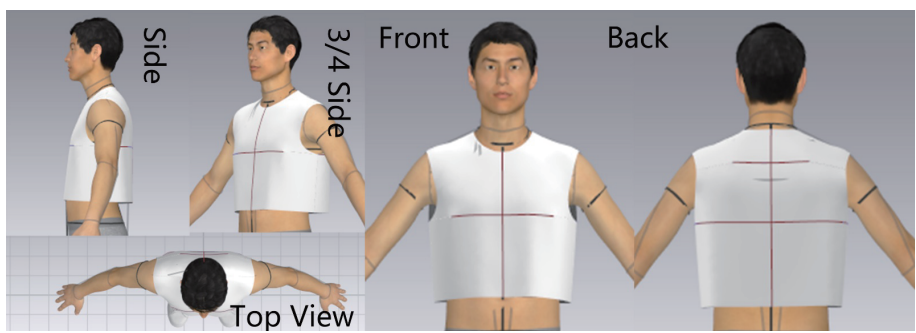

(a) Virtual try-on effect of the prototype

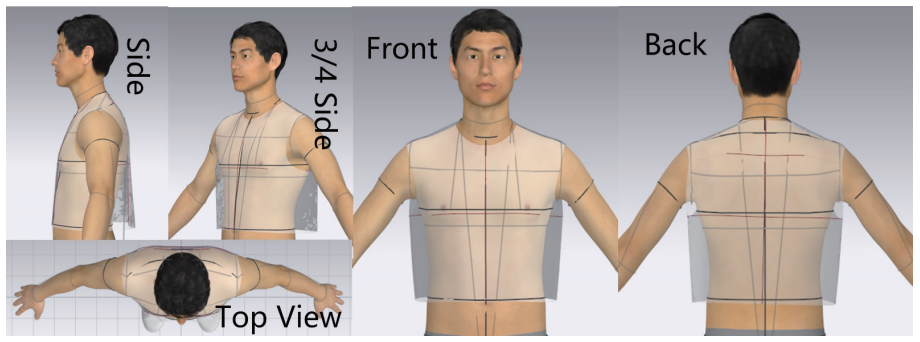

(b) Virtual try-on effect of the transparent pattern

Fig. 4: Virtual try-on effect of Donghua menswear prototype 
in order to tackle and solve them accordingly. In other words, each structural line can be solved independently and in parallel. After, the results are collected and combined, and we can get a complete directed graph model finally [17].

The fit analysis of the prototype's shoulder line was exemplified. Since the prototype shoulder angle is fixed and the length of the front shoulder line is determined by the back shoulder line, the pattern design model of the back shoulder line was extracted for analysis, as shown in Fig. 5.

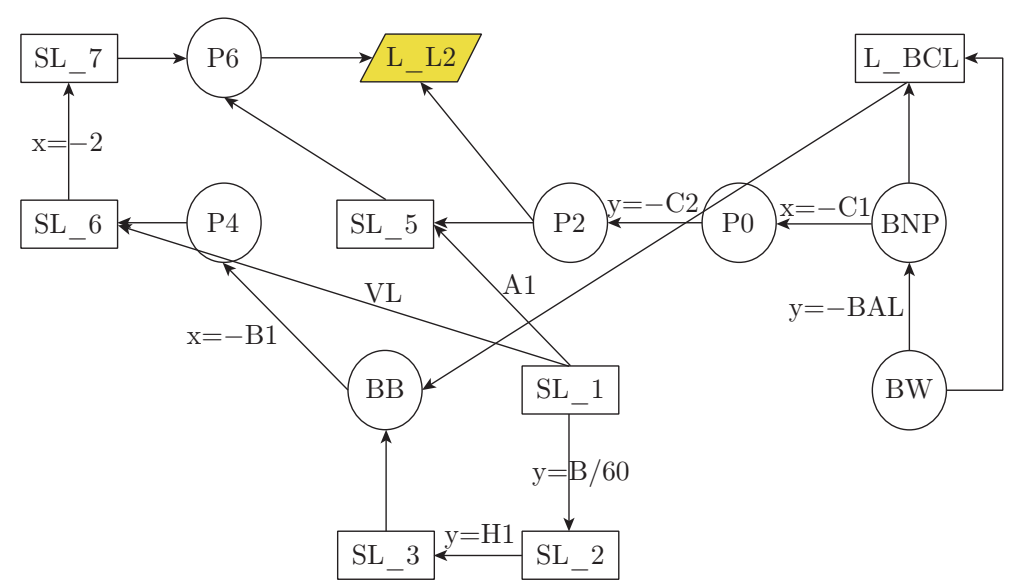

Fig. 5: The design model of the back shoulder line

As it can be seen from Fig. 5, The length of the front shoulder line L_L2 was mainly affected by the line SL_5, line SL_6 and the position of the point P2. Line SL_5 mainly affects the shape of the shoulder line, so the length of the shoulder line is determined by line SL_6 and the position of point P2. The straight-line SL_6 is subject to two constraints, one is the geometric constraint of the vertical relationship with the straight-line SL_1, and the other is the constraint of passing the point P4. Geometric constraints determine the state of line SL_4, point P4 determines the position of line SL_4, and the position of point P4 is determined by the back width B1 from point $\mathrm{BB}$ to point $\mathrm{P} 4$. The position of point $\mathrm{P} 2$ is determined by the distance $\mathrm{C} 2$ from point $\mathrm{P} 0$ to point $\mathrm{P} 2$, and $\mathrm{C} 2=\mathrm{C} 1 / 3$, so the final position of point $\mathrm{P} 2$ is determined by the back neck width $\mathrm{C} 1$. In summary, the length of the back shoulder line is ultimately determined by the back width B1 and the back neck width $\mathrm{C} 1$, and $\mathrm{B} 1=0.15 \mathrm{~B}+5.6 \mathrm{~cm}, \mathrm{C} 1=0.07 \mathrm{~B}+2 \mathrm{~cm}$, which are all calculated by the formula. This shows that the shoulder line doesn't fit. It can be seen that the root cause of the incompatibility of the shoulder line is that the application of the empirical formula cannot accurately determine the position of the back width line and the back neck width.

\subsection{Validation of the Fit Analysis Result of Donghua Menswear Pro- totype}

According to the above analysis of the fit of the shoulder line, the root cause of the lack of fit of the shoulder line is that the application of the empirical formula cannot accurately determine the position of the back width line and the back neck width. In order to verify the validity of the results of the fit analysis of the pattern design model, the back width and back neck width of the virtual model were measured to replace the calculation formula in the prototype, and then the prototype was made again according to the changed human data and the prototype design method. Then it was imported for virtual fitting in CLO 3D software and was compared with 
the previous fitting effect. According to the measurement, $\mathrm{B} 1^{\prime}=18.9 \mathrm{~cm}$ and $\mathrm{C} 1^{\prime}=7.5 \mathrm{~cm}$, then $\mathrm{C} 2^{\prime}=\mathrm{C} 1^{\prime} / 3=2.5 \mathrm{~cm}, \mathrm{C} 3^{\prime}=\mathrm{C} 1^{\prime}-0.3 \mathrm{~cm}=7.2 \mathrm{~cm}, \mathrm{C} 4^{\prime}=\mathrm{C} 1^{\prime}+0.5 \mathrm{~cm}=8 \mathrm{~cm}$. According to the modified data, the adjusted pattern was drawn, and after importing into CLO 3D software, the virtual fitting and the effect of the transparent pattern were shown in Fig. 6.

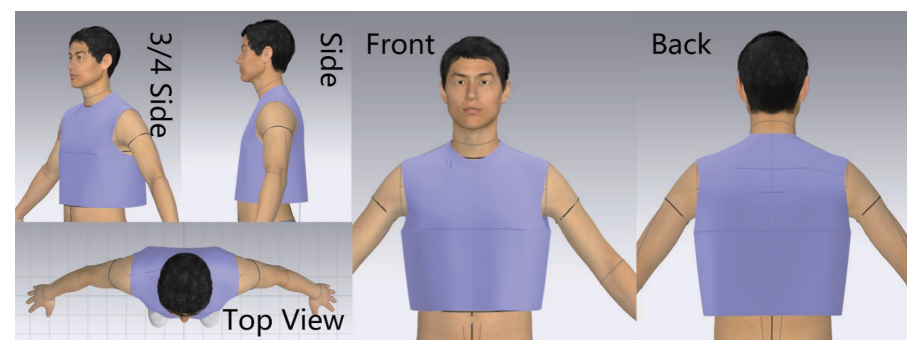

(a) Virtual try-on effect of the adjusted prototype

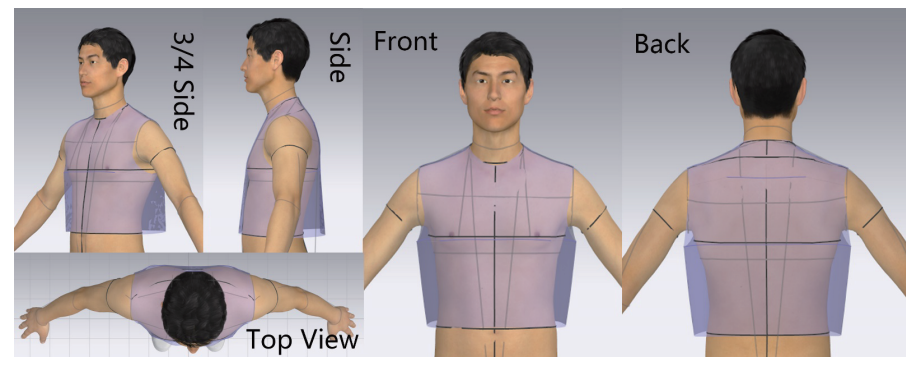

(b) Virtual try-on effect of the transparent pattern

Fig. 6: Virtual try-on effect of the adjusted prototype

As shown in Fig. 6, The adjusted neckline of the adjusted prototype fits well with the circumference of the neck of the human body, and the shoulder line fits well and is at the end of the shoulder.

The research shows that the pattern design model used to analyze the fit of clothing can find all the points, lines, constraints that affect the unfit parts of the clothing. It also traces the source of the problem and finds the root cause of the unfit parts, instead of adjusting the unfit parts simply. The experience of the designer in analyzing the pattern can be revealed by the method. The latter can avoid the imbalance of the pattern caused by simply adjusting the structure of the unfit parts of the clothing.

\section{Results}

Following Donghua menswear prototype as illustrated by the research, the prototype design model of the prototype was constructed by the directed graph method, the fit of the prototype was analyzed using the model and the validity of the analysis results was verified. The research results indicate that the use of the directed graph method to build a clothing pattern design model can clearly show all the points, line elements, the constraint relationship between each element and the process of pattern making required in a graphical way. The model enables the knowledge of pattern design to be collected and sorted out, making the experience of the pattern maker explicit; using the model to analyze the pattern is not only clear and accurate, but also can be traced to the source to understand the specific factors and root causes that affect the pattern. This research can provide a way to collect, extract, process, share and reuse pattern 
design knowledge for intelligent clothing design, and improve the efficiency of clothing pattern design.

\section{References}

[1] Zhou J. Intelligent_—-Main Direction of "Made in China 2025". China Mechanical Engineering: 2015; 26 (17): 2273-84.

[2] Wang FY, Wang X, Wang S. Knowledge automation: the foundation and key of intelligent industry. Zhang Jiang Technology Review: 2017; 1: 35-38.

[3] Gui WH, Chen XF, Yang CH, Xie YF. Knowledge automation and its industrial application. SCIENTIA SINICA (Informationis): 2016; 46 (08): 1016-1034.

[4] Liu YD. Research and application of intelligent method for garment pattern generation - men's shirt smart pattern generation. Soochow University: 2019.

[5] Liu WM, Xie H. Generation of intelligent fitting pattern based on BP neural network. Journal of Textile Research: 2018; 39 (07): 116-121.

[6] Liu F, Luo RL, Fan ML. Automatic generation of the pattern size of tailored suit. Wool Textile Journal: 2019; 47 (02): 50-53.

[7] Zhou HJ. Research on Automatic Pattern Making Rules of Female Trousers Based on 3D Measurement Data. Donghua University: 2018.

[8] Mesuda Y, Inui S, Horiba Y. Virtual draping by mapping. Computers in Industry: 2018; 95 : 93-101.

[9] Ye QW, Zhang GP. Generation of personalized garment pattern based on Auto CAD parameterization. Journal of Textile Research: 2019; 40 (4): 103-110.

[10] Zhang LL, Zhang GP. Parametric flat pattern design for clothing based on Mat Lab. Journal of Textile Research: 2019; 40 (01): 130-135.

[11] Han HS, Kim S, Park CK. Automatic custom pattern generation using width-height independent grading. International Journal of Clothing Science and Technology: 2015; 27 (6): 908-21.

[12] Harwood ARG, Gill J, Gill S. JBlockCreator: An open source, pattern drafting framework to facilitate the automated manufacture of made-to-measure clothing. Software X: 2020; 11: 100365.

[13] Zhou WC. Construction of Intelligent Clothing Pattern Model Based on Domain Ontology. Xi'an Polytechnic University: 2016.

[14] Kang WW. Research and Implementation of Rocket Fault Diagnosis Technology Based on Directed Graph and Fuzzy Set. Lanzhou University: 2016.

[15] Huang CS. Power system fault layered abductive analysis based on directed graph and fault tree. Southwest Jiaotong University: 2014.

[16] Zhang HZ. Garment CAD theory and application. China Textile \& Apparel Press: 2005; p.141.

[17] Ying BA, Guo HJ, Zhang X, Qi J. A method of constructing clothing pattern design model by directed graphs. CHN Patent No.201810846721.2: 2018. 